\title{
An Arc Search Interior-Point Algorithm for Monotone Linear Complementarity Problems over Symmetric Cones
}

\section{Mohammad Pirhaji ${ }^{a}$, Maryam Zangiabadi ${ }^{a}$, Hossein Mansouri ${ }^{a}$ and Saman H. Amin ${ }^{b}$}

${ }^{a}$ Department of Applied Mathematics, Shahrekord University Shahrekord, Iran

${ }^{b}$ Department of Mechanical and Industrial Engineering, Ryerson University

Toronto, Canada

E-mail(corresp.): zangiabadi-m@sci.sku.ac.ir

Received December 10, 2016; revised December 11, 2017; accepted December 11, 2017

\begin{abstract}
An arc search interior-point algorithm for monotone symmetric cone linear complementarity problem is presented. The algorithm estimates the central path by an ellipse and follows an ellipsoidal approximation of the central path to reach an $\varepsilon$-approximate solution of the problem in a wide neighborhood of the central path. The convergence analysis of the algorithm is derived. Furthermore, we prove that the algorithm has the complexity bound $O(\sqrt{r} L)$ using Nesterov-Todd search direction and $O(r L)$ by the $x s$ and $s x$ search directions. The obtained iteration complexities coincide with the best-known ones obtained by any proposed interiorpoint algorithm for this class of mathematical problems.
\end{abstract}

Keywords: linear complementarity problem, symmetric cone, ellipsoidal approximation, interior-point methods, polynomial complexity.

AMS Subject Classification: 90C33; 90C51.

\section{Introduction}

Let $(\mathcal{V}, \circ)$ be an $n$-dimensional Euclidean Jordan algebra (EJA) with rank $r$ equipped with the standard inner product $\langle x, s\rangle:=\operatorname{tr}(x \circ s)$ and assume that $\mathcal{K}$ is the symmetric cone related to $\operatorname{EJA}(\mathcal{V}, \circ)$. In this paper, similar to $[8,9,22]$, we define the monotone symmetric cone linear complementarity problem (SCLCP) in the standard form as follows: The problem of finding a pair $(x, s) \in \mathcal{K} \times \mathcal{K}$

Copyright (c) 2018 The Author(s). Published by VGTU Press

This is an Open Access article distributed under the terms of the Creative Commons Attribution License (http://creativecommons.org/licenses/by/4.0/), which permits unrestricted use, distribution, and reproduction in any medium, provided the original author and source are credited. 
such that

$$
s=\mathcal{M}(x)+q, \quad x \circ s=0,
$$

where $q \in \mathcal{V}$ and $\mathcal{M}: \mathcal{V} \longrightarrow \mathcal{V}$ is a positive semidefinite linear operator. That is, $\langle\mathcal{M}(x), x\rangle \geq 0$ for $x \in \mathcal{V}$. Since $\mathcal{V}$ is EJA, we can consider the matrix representation $\mathcal{M}(x)=M x$ in which $M \in \mathbb{R}^{n \times n}$ is positive semidefinite with respect to the inner product $\langle\cdot, \cdot\rangle$ in $(\mathcal{V}, \circ)$. Therefore, the above problem can be reformulated as follows:

$$
s=M x+q, \quad x \circ s=0 .
$$

The monotone SCLCP is a general class of mathematical problems which contains the monotone LCP (the monotone SCLCP with $\mathcal{K}=\mathbb{R}_{+}^{n}$ (the cone of nonnegative orthant)), the monotone semidefinite LCP (the monotone SCLCP with $\mathcal{K}=\mathbb{S}_{+}^{n}$ (the cone of positive semidefinite matrices)) and the monotone second-order cone LCP (the monotone SCLCP with $\mathcal{K}=\mathcal{L}_{+}^{n}$ (the second-order cone)). Among various approaches, interior-point methods (IPMs) are quite efficient for solving various classes of mathematical problems. Since Karmarkar's algorithm [7] was proved to be polynomial, many researchers proposed some interior-point algorithms for solving mathematical problems over non-negative orthant.

Using EJA, Faybusovich [4] generalized IPMs over non-negative orthant problems to symmetric optimization (SO) problems and SCLCPs. Gowda and Sznajder [5] showed some global uniqueness and solvability result for SCLCPs. Schemita and Alizadeh [17] proved the convergence analysis and the polynomial iteration-complexity for short, semi-long and long-step interior-point algorithms for SO problems using the commutative class of search directions. Rangarajan [15] proposed the first infeasible interior-point method (IIPM) over symmetric cones. Yoshise [21] proposed a homogeneous algorithm for monotone nonlinear symmetric cone complementarity problems. Gu et al. [23] extended the proposed algorithm by Roos [16] for linear optimization (LO) to SO problems. Wang et al. [22] generalized the $\mathrm{Gu}$ et al.'s feasible algorithm for SO [6] to the Cartesian $P_{*}(\mathcal{K})$-SCLCP and obtained the complexity bound $O(\sqrt{r} L)$ for their algorithm.

In most of the above mentioned papers, the proposed algorithms search an $\varepsilon$-approximate solution of the underlying problem in a small neighborhood of a straight line related to the first and second-order derivatives of the central path. However, the most mathematical problems are nonlinear and it seems that the perfect search for obtaining an $\varepsilon$-approximate solution of them is not along a straight line and it is along the arcs. Therefore, it is worth to investigate the analysis of some interior-point algorithms that follow an arc instead of a straight line in procedure of finding an $\varepsilon$-approximate solution of the underlying problems.

The first arc search interior-point algorithms were suggested by Yang $[19,20]$ for convex quadratic optimization (CQO) problems and LO problems. These algorithms utilize the first and second-order derivatives to construct an ellipse for approximating the central path. Searching along the ellipse is more efficient 
than searching along any straight line due to generating a larger step size. This makes the Yang's algorithms [19,20] efficient in practical computation.

$\mathrm{Ai}$ and Zhang [1] based on a new wide neighborhood of the central path, proposed the first wide neighborhood interior-point algorithm for LCPs. After that, several authors generalized the Ai and Zhang's algorithm for LCPs [1] to some other problems. For instance, Li and Terlaky [10] extended the Ai and Zhang's algorithm for LCPs [1] to semidefinite optimization problems. Yang et al. [13], based on Ai and Zhang's wide neighborhood, proposed a new infeasible IPM for SO problems.

Motivated by Yang [19,20], Yang et al. [25] proposed a wide neighborhood arc search infeasible interior-point algorithm for LO problems. Pirhaji et al. [24], using the arc search strategy and the wide neighborhood given by Ai and Zhang [1], suggested an infeasible interior-point algorithm for monotone LCPs. Based on a commutative class of search directions and the Ai-Zhang's wide neighborhood, Yang et al. [14] generalized their proposed arc search algorithm for LO problems [25] to SO problems.

The main goal of this paper is to present a wide neighborhood arc search feasible interior-point algorithm for monotone SCLCPs. To this end, motivated by Yang $[19,20]$, we first estimate the central path of monotone SCLCP by an ellipse and then, using the wide neighborhood given by Ai and Zhang [1], we propose an arc search feasible interior-point algorithm for monotone SCLCPs. The algorithm obtains an $\varepsilon$-approximate solution of the monotone SCLCP in $O(\sqrt{r} L)$ iterations using Nesterov-Todd search direction and it will terminate after $O(r L)$ iterations, using the $x s$ and $s x$ search directions.

The paper is organized as follows. In Section 2, we recall some basic concepts on EJAs which are required in our paper. In Section 3, we first introduce the central path of the SCLCP and its ellipsoidal approximation, then we propose an arc search feasible interior-point algorithm for solving monotone SCLCPs. Section 4 is devoted to present some technical lemmas which are required in convergence analysis of the algorithm. The convergence analysis of the algorithm will be presented in Subsection 4.2. Finally, the paper ends with some concluding remarks in Section 5 .

\section{$2 \quad$ Preliminaries}

In this section, we outline a minimal foundation of the theory of EJAs which will be used in this paper.

The $\operatorname{EJA}(\mathcal{V}, \circ)$ is a finite dimensional vector space over $\mathbb{R}$ equipped with the bilinear map $\circ:(x, y) \longrightarrow x \circ y \in \mathcal{V}$ and the standard inner product $\langle x, s\rangle:=$ $\operatorname{tr}(x \circ s)$. The related cone of squares corresponding with $(\mathcal{V}, \circ)$ is called the symmetric cone $\mathcal{K}$. For each $x, y \in \mathcal{V}, L_{x} y:=x \circ y$ and $Q_{x}:=2 L_{x}^{2}-L_{x^{2}}$, where $L_{x}^{2}:=L_{x} L_{x}$, denote the linear and quadratic representation of $\mathcal{V}$, respectively.

A Jordan algebra has an identity element, if there exists a unique element $e \in \mathcal{V}$ such that $x \circ e=e \circ x=x$ for all $x \in \mathcal{V}$. An element $c \in \mathcal{V}$ is said to be idempotent if $c^{2}=c$. An idempotent $c$ is primitive if it is nonzero and can not be expressed by sum of two other nonzero idempotents. A set of idempotents $\left\{c_{1}, c_{2}, \ldots, c_{k}\right\}$ is called a Jordan frame if $c_{i} \circ c_{j}=0$ for any $i \neq j$, 
and $\sum_{i=1}^{k} c_{i}=e$. For any $x \in \mathcal{V}$, let $l$ be the smallest positive integer such that $\left\{e, x, x^{2}, \ldots, x^{l}\right\}$ is linearly dependent, $l$ is called the degree of $x$ and is denoted by $\operatorname{deg}(x)$. The rank of $\mathcal{V}$, denoted by $\operatorname{rank}(\mathcal{V})$, is defined as the maximum of $\operatorname{deg}(x)$ over all $x \in \mathcal{V}$.

The spectral decomposition theorem (Theorem III.1.2 in [3]) of a Euclidean Jordan algebra $\mathcal{V}$ states that for any $x \in \mathcal{V}$ there exists a Jordan frame $\left\{c_{1}, c_{2}, \ldots, c_{k}\right\}$ and a set of real numbers $\left\{\lambda_{1}, \lambda_{2}, \ldots, \lambda_{k}\right\}$ (the eigenvalues of $x)$ such that $x=\sum_{i=1}^{k} \lambda_{i} c_{i}$. For any $x \in \mathcal{V}$, we define $\operatorname{tr}(x):=\sum_{i=1}^{k} \lambda_{i}$ and $\operatorname{det}(x):=\prod_{i=1}^{k} \lambda_{i}$. We also define $\lambda_{\min }(x)$ and $\lambda_{\max }(x)$ as the minimum and maximum eigenvalue of $x$, respectively. The norm induced by the standard inner product is named as the Frobenius norm, which is given by $\|x\|_{F}:=\sqrt{\langle x, x\rangle}$. Some other norms related to the absolute value of eigenvalues of $x$, namely 1-norm and infinity norm, are defined as $\|x\|_{1}:=\sum_{i=1}^{k}\left|\lambda_{i}(x)\right|$ and $\|x\|_{2}:=\max _{i}\left|\lambda_{i}(x)\right|$. Moreover, the merit projection $x^{+}:=\sum_{i=1}^{r} \lambda_{i}^{+} c_{i}$ and $x^{-}:=x-x^{+}$, where $\lambda_{i}^{+}:=\max \left\{\lambda_{i}, 0\right\}$ for $i=1,2, \ldots, k$.

\section{Central path and its ellipsoidal approximation}

In this section, we first define the central path of monotone SCLCP and then we estimate it by an ellipse. To this end, let

$$
\begin{aligned}
& F:=\{(x, s) \in \mathcal{K} \times \mathcal{K}: \quad s=M x+q\}, \\
& F^{0}:=\{(x, s) \in \operatorname{int} \mathcal{K} \times \operatorname{int} \mathcal{K}: \quad s=M x+q\},
\end{aligned}
$$

respectively denote the feasible and strictly feasible sets of monotone SCLCP. Here, we assume $F^{0} \neq \emptyset$. That is, problem (1.1) satisfies the interior-point condition (IPC).

The main idea of feasible IPMs is to replace the complementarity condition $x \circ s=0$ in (1.1) by the perturbed equation $x \circ s=\mu e$ to get the following parameterized system:

$$
M x-s+q=0, \quad x \circ s=\mu e .
$$

Considering a scaling point $p \in \mathcal{C}(x, s)$, where

$$
\mathcal{C}(x, s):=\left\{p \mid p \text { nonsingular, } Q_{p} x \text { and } Q_{p^{-1}} s \text { operator commute }\right\}
$$

defining

$$
\widetilde{M}:=Q_{p^{-1}} M Q_{p^{-1}}, \quad \tilde{x}:=Q_{p} x, \quad \tilde{s}:=Q_{p^{-1}} s, \quad \tilde{q}:=Q_{p^{-1}} q
$$

and using Lemma 28 in [17], system (3.1) can be rewritten as follows:

$$
\widetilde{M} \tilde{x}-\tilde{s}+\tilde{q}=0, \quad \tilde{x} \circ \tilde{s}=\mu e .
$$

Some best-known choices for the scaling vector $p$, such as $p:=x^{-\frac{1}{2}}$ and $p:=s^{\frac{1}{2}}$, have been proposed by some researchers. These choices of the scaling vector 
$p$ respectively led to the well-known search directions $x s$ and $s x$ while for the choice of

$$
p:=\left(Q_{x^{\frac{1}{2}}}\left(Q_{x^{\frac{1}{2}}} s\right)^{-\frac{1}{2}}\right)^{-\frac{1}{2}}=\left(Q_{s^{-\frac{1}{2}}}\left(Q_{s^{\frac{1}{2}}} x\right)^{\frac{1}{2}}\right)^{-\frac{1}{2}}
$$

we get the Nesterov-Todd (NT) search direction. System (3.3) has a unique solution $(\tilde{x}(\mu), \tilde{s}(\mu))$ for any $\mu>0$. The set of all such solutions gives a homotopy path, which is called the central path. Mathematically, the central path is a parameterized function of $\mu$ defined as

$$
\mathcal{H}(\mu):=\{(\tilde{x}(\mu), \tilde{s}(\mu)):(\tilde{x}(\mu), \tilde{s}(\mu)) \text { satisfies }(3.3) \text { with } \mu>0\} .
$$

Clearly, the most efficient way to find an $\varepsilon$-approximate solution of the monotone SCLCP is to follow the central path while decreasing the parameter $\mu$ to zero. If $\mu \rightarrow 0$, then the limit of the central path exists and it is an $\varepsilon$ approximate solution of the monotone SCLCP.

As we mentioned before, most of interior-point algorithms follow a straight line approximation related to the first and second order derivatives of the central path and generate a sequence of iterations in a small neighborhood of the central path. In this paper, we use the neighborhood

$$
\mathcal{N}(\tau, \beta):=\left\{(x, s) \in \mathcal{F}^{0}:\left\|\left(\tau \mu e-Q_{x^{\frac{1}{2}}} s\right)^{+}\right\|_{F} \leq \beta \tau \mu\right\}
$$

of the central path, where $\beta, \tau \in[0,1]$. Motivated by Yang $[19,20]$, we estimate the central path $\mathcal{H}(\mu)$ by the ellipse $\xi(\alpha) \in \mathbb{R}^{2 n}[2]$ which is defined as follows:

$$
\xi(\alpha):=\left\{(\tilde{x}(\alpha), \tilde{s}(\alpha)) \in \mathbb{R}^{2 n}:(\tilde{x}(\alpha), \tilde{s}(\alpha))=\overrightarrow{\mathbf{a}} \cos (\alpha)+\overrightarrow{\mathbf{b}} \sin (\alpha)+\overrightarrow{\mathbf{c}}\right\}
$$

where $\overrightarrow{\mathbf{a}}$ and $\overrightarrow{\mathbf{b}} \in \mathbb{R}^{2 n}$ are the axes of the ellipse which are perpendicular to each other and $\overrightarrow{\mathbf{c}} \in \mathbb{R}^{2 n}$ is the center of the ellipse.

Let $(\tilde{x}, \tilde{s})=\left(\tilde{x}\left(\alpha_{0}\right), \tilde{s}\left(\alpha_{0}\right)\right) \in \xi(\alpha)$ be close to or on the central path $\mathcal{H}(\mu)$. We proceed to determine the vectors $\overrightarrow{\mathbf{a}}, \overrightarrow{\mathbf{b}}, \overrightarrow{\mathbf{c}} \in \mathbb{R}^{2 n}$ and the angle $\alpha_{0}$ such that the first and second derivatives of $\xi(\alpha)$ at $(\tilde{x}, \tilde{s})$ are the same as those of $\mathcal{H}(\mu)$ at $(\tilde{x}, \tilde{s})$. Therefor, defining $(\dot{\tilde{x}}, \dot{\tilde{s}})$ and $(\ddot{\tilde{x}}, \ddot{\tilde{s}})$ as the first and second derivatives of $(\tilde{x}, \tilde{s})$ and taking the first and second derivatives of $(3.3)$ at $(\tilde{x}, \tilde{s})$ with respect to $\mu$, we obtain

$$
\begin{array}{ll}
\widetilde{M} \dot{\tilde{x}}-\dot{\tilde{s}}=0, & \dot{\tilde{x}} \circ \tilde{s}+\tilde{x} \circ \dot{\tilde{s}}=e, \\
\widetilde{M} \ddot{\tilde{x}}-\ddot{\tilde{s}}=0, & \ddot{\tilde{x}} \circ \tilde{s}+2 \dot{\tilde{x}} \circ \dot{\tilde{s}}+\tilde{x} \circ \ddot{\tilde{s}}=0 .
\end{array}
$$

In this paper, motivated by [14] and [25], we define the first and second derivatives at $(\tilde{x}, \tilde{s})$ to satisfy

$$
\begin{array}{ll}
\widetilde{M} \dot{\tilde{x}}-\dot{\tilde{s}}=0, & \tilde{s} \circ \dot{\tilde{x}}+\tilde{x} \circ \dot{\tilde{s}}=\tilde{r}_{c}, \\
\widetilde{M} \ddot{\tilde{x}}-\ddot{\tilde{s}}=0, \quad \tilde{s} \circ \ddot{\tilde{x}}+\tilde{x} \circ \ddot{\tilde{s}}=-2 \dot{\tilde{x}} \circ \dot{\tilde{s}}
\end{array}
$$


where $\tilde{r}_{c}:=-\left[(\tau \mu e-\tilde{x} \circ \tilde{s})^{-}+\sqrt{r}(\tau \mu e-\tilde{x} \circ \tilde{s})^{+}\right]$. The reason for choosing $\tilde{r}_{c}$ in equation (3.7) is that the extra computational effort is marginal compared to the computation of Newton search directions with choosing any classical terms such as $e$ and $\tau \mu e-\tilde{x} \circ \tilde{s}$ (see $[14,18,25])$.

Obviously, since $M$ is a positive semidefinite matrix $\widetilde{M}$ is also a positive semidefinite one. Let $\alpha \in\left[0, \frac{\pi}{2}\right]$. The following lemma denotes the mathematical form of the ellipsoidal approximation of the central path $\mathcal{H}(\mu)$.

Lemma 1 [Lemma 3.1 in [19]]. Let $\xi(\alpha)$ be the defined ellipse in (3.4) which passes a point $(\tilde{x}, \tilde{s})$. Moreover, assume that the first and second derivatives $(\dot{\tilde{x}}, \dot{\tilde{s}})$ and $(\ddot{\tilde{x}}, \ddot{\tilde{s}})$ satisfy (3.5) and (3.6). Then, after searching along the ellipse $\xi(\alpha)$, the new generated point $(\tilde{x}(\alpha), \tilde{s}(\alpha))$ is given by

$$
\begin{aligned}
& \tilde{x}(\alpha):=\tilde{x}-\dot{\tilde{x}} \sin (\alpha)+\ddot{\tilde{x}}(1-\cos (\alpha)), \\
& \tilde{s}(\alpha):=\tilde{s}-\dot{\tilde{s}} \sin (\alpha)+\ddot{\tilde{s}}(1-\cos (\alpha)) .
\end{aligned}
$$

Due to the above lemma, the ellipsoidal approximation of the central path $\mathcal{H}(\mu)$ is mathematically defined as follows:

$$
\widetilde{\mathcal{H}}(\alpha):=\left\{(\tilde{x}(\alpha), \tilde{s}(\alpha)):(\tilde{x}(\alpha), \tilde{s}(\alpha)) \text { satisfies (3.7) and (3.8) with } \alpha \in\left[0, \frac{\pi}{2}\right]\right\} \text {. }
$$

However, after searching along the ellipsoidal central path $\widetilde{\mathcal{H}}(\alpha)$, the new generated point $(\tilde{x}(\alpha), \tilde{s}(\alpha))$ is given by Lemma 1 . Defining $g(\alpha):=1-\cos (\alpha)$, we have

$$
\begin{aligned}
& \tilde{x}(\alpha) \circ \tilde{s}(\alpha)=\tilde{x} \circ \tilde{s}-\sin (\alpha) \tilde{r}_{c}+\varphi(\alpha), \\
& \varphi(\alpha):=-g^{2}(\alpha) \dot{\tilde{x}} \circ \dot{\tilde{s}}-\sin (\alpha) g(\alpha)(\dot{\tilde{x}} \circ \ddot{\tilde{s}}+\dot{\tilde{s}} \circ \ddot{\tilde{x}})+g^{2}(\alpha) \ddot{\tilde{x}} \circ \ddot{\tilde{s}}
\end{aligned}
$$

\subsection{Arc search algorithm}

Here, the main idea of arc search feasible interior-point algorithm for monotone SCLCP is described. The arc search algorithm starts from an initial point $\left(x^{0}, s^{0}\right) \in \mathcal{N}(\tau, \beta)$ which is close to or on the central path $\mathcal{H}(\mu)$. Constructing an ellipse which passes through this point, the central path $\mathcal{H}(\mu)$ is approximated. Then, instead of the central path $\mathcal{H}(\mu)$, the algorithm follows the ellipsoidal approximation $\widetilde{\mathcal{H}}(\alpha)$ to get a new iterate $(\tilde{x}(\alpha), \tilde{s}(\alpha))$. The new iterate $(\tilde{x}(\alpha), \tilde{s}(\alpha))$ not only reduces the duality gap $\operatorname{tr}(\tilde{x} \circ \tilde{s})$ but also belongs to the wide neighborhood $\mathcal{N}(\tau, \beta)$. This procedure will be repeated until an $\varepsilon$-approximate solution of the monotone SCLCP is found.

Below, a more formal description of the algorithm is presented.

Algorithm 1. (Arc search feasible interior-point algorithm for monotone SCLCP)

- Input parameters: An accuracy parameter $\varepsilon>0$, a neighborhood parameter $\beta \in\left(0, \frac{1}{2}\right]$, a centering parameter $\tau \in\left(0, \frac{1}{4}\right]$, an initial feasible point $\left(x^{0}, s^{0}\right) \in \mathcal{N}(\tau, \beta)$ and $\mu^{0}=\frac{1}{r} \operatorname{tr}\left(x^{0} \circ s^{0}\right)$.

- Step 0: Set $\mathrm{k}=0,1,2, \ldots$. 
- Step 1: If $\tilde{\mu}^{k} \leq \varepsilon \tilde{\mu}^{0}$, stop. Otherwise, go to the next step.

- Step 2: Compute $(\dot{\tilde{x}}, \dot{\tilde{s}}),(\ddot{\tilde{x}}, \ddot{\tilde{s}})$ respectively by the systems $(3.5)$ and (3.6).

- Step 3: Calculate the largest step $\operatorname{size} \sin \left(\hat{\alpha}^{k}\right)$ such that

$$
\begin{aligned}
& \tilde{\mu}(\alpha) \leq(1-0.5 \sin (\alpha)) \tilde{\mu}^{k}, \\
& (\tilde{x}(\alpha), \tilde{s}(\alpha)) \in \mathcal{N}(\tau, \beta),
\end{aligned}
$$

for any step $\operatorname{size} \sin (\alpha) \in\left[0, \sin \left(\hat{\alpha}^{k}\right)\right]$.

- Step 4: Compute the new iterate $\left(\tilde{x}\left(\hat{\alpha}^{k}\right), \tilde{s}\left(\hat{\alpha}^{k}\right)\right)$ by Lemma 1 and $\tilde{\mu}\left(\hat{\alpha}^{k}\right):=$ $\operatorname{tr}\left(\tilde{x}\left(\hat{\alpha}^{k}\right) \circ \tilde{s}\left(\hat{\alpha}^{k}\right)\right) / r$.

- Step 5: Set $\tilde{\mu}^{k+1}=\tilde{\mu}\left(\hat{\alpha}^{k}\right)$ and $k=k+1$ and go to Step 1 .

\section{Complexity analysis of the algorithm}

\subsection{Technical lemmas}

In this subsection, we state some technical lemmas which are required in proof of the convergence analysis of the algorithm.

Lemma 2. The neighborhood $\mathcal{N}(\tau, \beta)$ is scaling invariant, i.e., $(x, s)$ belongs to the neighborhood $\mathcal{N}(\tau, \beta)$ iff $(\tilde{x}, \tilde{s})$ does.

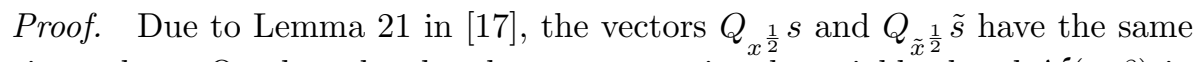
eigenvalues. On the other hand, we can rewrite the neighborhood $\mathcal{N}(\tau, \beta)$ in term of the eigenvalues of $Q_{x^{\frac{1}{2}}} s$. This follows the result.

Lemma 3. Let $x, s \in$ int $\mathcal{K}$. If $x$ and $s$ are operator commute, then $Q_{x^{\frac{1}{2}}} s=$ $x \circ s$.

Proof. Let $x$ and $s$ be operator commute. Then, $x^{\frac{1}{2}}$ and $s$ are also operator commute. This implies

$$
\begin{aligned}
Q_{x^{\frac{1}{2}}} s & =\left[2 L\left(x^{\frac{1}{2}}\right)^{2}-L(x)\right] s=2 L\left(x^{\frac{1}{2}}\right)^{2} L(s) e-x \circ s \\
& =2 L(s) L\left(x^{\frac{1}{2}}\right)^{2} e-x \circ s=2 L(s) x-x \circ s=x \circ s .
\end{aligned}
$$

This completes the proof.

Corollary 1. Let $x, s \in$ int $\mathcal{K}$ and $\tilde{x}$ and $\tilde{s}$ be as defined in (3.2). Then, $Q_{\tilde{x}^{\frac{1}{2}}} \tilde{s}=$ $\tilde{x} \circ \tilde{s}$.

Proof. Due to Lemma 2.2 in [11], if $x, s \in$ int $\mathcal{K}$ then we have $\tilde{x}, \tilde{s} \in$ int $\mathcal{K}$. Moreover, $\tilde{x}$ and $\tilde{s}$ are operator commute. The result directly follows by Lemma 3.

The following lemma is a direct result of the positive semidefinite property of the matrix $\widetilde{M}$ in systems (3.5) and (3.6). 
Lemma 4. Let $(\dot{\tilde{x}}, \dot{\tilde{s}})$ and $(\ddot{\tilde{x}}, \ddot{\tilde{s}})$ respectively be the solutions of systems (3.5) and (3.6). Then

$$
\operatorname{tr}(\dot{\tilde{x}} \circ \dot{\tilde{s}}) \geq 0, \quad \operatorname{tr}(\ddot{\tilde{x}} \circ \ddot{\tilde{s}}) \geq 0 .
$$

Lemma 5 [Lemma 5.3 in [18]]. Let $(\tilde{x}, \tilde{s}) \in \mathcal{N}(\tau, \beta)$ and $G:=L_{\tilde{s}}^{-1} L_{\tilde{x}}$. Then,

$$
\left\|\left(L_{\tilde{x}} L_{\tilde{s}}\right)^{-\frac{1}{2}} \tilde{r}_{c}\right\|_{F}^{2} \leq(1+\beta \tau) \mu r .
$$

Lemma 6. Let $(\tilde{x}, \tilde{s}) \in \mathcal{N}(\tau, \beta), G:=L_{\tilde{s}}^{-1} L_{\tilde{x}}$ and $(\dot{\tilde{x}}, \dot{\tilde{s}})$ be the solution of system (3.5). Then,

$$
\begin{aligned}
\|\dot{\tilde{x}} \circ \dot{\tilde{s}}\|_{F} & \leq 0.5 \sqrt{\operatorname{cond}(G)}(1+\beta \tau) \mu r \\
\operatorname{tr}(\dot{\tilde{x}} \circ \dot{\tilde{s}}) & \leq 0.5 \sqrt{\operatorname{cond}(G)}(1+\beta \tau) \mu r .
\end{aligned}
$$

Proof. Multiplying the second equation in system (3.5) by $\left(L_{\tilde{x}} L_{\tilde{s}}\right)^{-\frac{1}{2}}$, taking squared norm on both sides and using Lemma 33 in [17] and Lemmas 4 and 5, we derive

$$
\begin{aligned}
& \|\dot{\tilde{x}} \circ \dot{\tilde{s}}\|_{F} \leq\|\dot{\tilde{x}}\|_{F}\|\dot{\tilde{s}}\|_{F} \leq 0.5 \sqrt{\operatorname{cond}(G)}\left(\left\|G^{\frac{-1}{2}} \dot{\tilde{x}}\right\|_{F}^{2}+\left\|G^{\frac{1}{2}} \dot{\tilde{s}}\right\|_{F}^{2}\right) \\
& \quad \leq 0.5 \sqrt{\operatorname{cond}(G)}\left\|G^{\frac{-1}{2}} \dot{\tilde{x}}+G^{\frac{1}{2}} \dot{\tilde{s}}\right\|_{F}^{2}=0.5 \sqrt{\operatorname{cond}(G)}\left\|\left(L_{\tilde{x}} L_{\tilde{s}}\right)^{-\frac{1}{2}} \tilde{r}_{c}\right\|_{F}^{2} \\
& \quad \leq 0.5 \sqrt{\operatorname{cond}(G)}(1+\beta \tau) \mu r
\end{aligned}
$$

which implies (4.1). To prove (4.2), using Lemma 4.52 in [6], in the same way as the proof of (4.1), we obtain

$$
\operatorname{tr}(\dot{\tilde{x}} \circ \dot{\tilde{s}}) \leq\|\dot{\tilde{x}}\|_{F}\|\dot{\tilde{s}}\|_{F} \leq \frac{1}{2} \sqrt{\operatorname{cond}(G)}(1+\beta \tau) \mu r .
$$

This completes the proof.

Lemma 7. Let $\gamma:=(1-\beta) \tau,(\tilde{x}, \tilde{s}) \in \mathcal{N}(\tau, \beta), G:=L_{\tilde{s}}^{-1} L_{\tilde{x}}$ and $(\ddot{\tilde{x}}, \ddot{\tilde{s}})$ be the solution of system (3.6). Then,

$$
\left\|G^{\frac{-1}{2}} \ddot{\tilde{x}}+G^{\frac{1}{2}} \ddot{\tilde{s}}\right\|_{F}^{2} \leq \frac{1}{\gamma} \operatorname{cond}(G)(1+\beta \tau)^{2} \mu r^{2} .
$$

Proof. Multiplying the second equation in system (3.6) by $\left(L_{\tilde{x}} L_{\tilde{s}}\right)^{-\frac{1}{2}}$, taking squared norm on both sides and using Lemma 6, we have

$$
\begin{aligned}
\| G^{\frac{-1}{2} \ddot{\tilde{x}}+G^{\frac{1}{2}} \ddot{\tilde{s}} \|_{F}^{2}} & =4\left\|\left(L_{\tilde{x}} L_{\tilde{s}}\right)^{-\frac{1}{2}} \dot{\tilde{x}} \circ \dot{\tilde{s}}\right\|_{F}^{2}=4\left\|\left(L_{\tilde{x}} L_{\tilde{s}}\right)^{-\frac{1}{2}}\right\|_{2}^{2}\|\dot{\tilde{x}} \circ \dot{\tilde{s}}\|_{F}^{2} \\
& =4 \lambda_{\max }\left(L_{\tilde{x}} L_{\tilde{s}}\right)^{-1}\|\dot{\tilde{x}} \circ \dot{\tilde{s}}\|_{F}^{2}=\frac{4}{\lambda_{\min }(\tilde{x} \circ \tilde{s})}\|\dot{\tilde{x}} \circ \dot{\tilde{s}}\|_{F}^{2} \\
& \leq \frac{4}{\gamma \mu}\|\dot{\tilde{x}} \circ \dot{\tilde{s}}\|_{F}^{2} \leq \frac{1}{\gamma} \operatorname{cond}(G)(1+\beta \tau)^{2} \mu r^{2},
\end{aligned}
$$

where, the first inequality follows from $(\tilde{x}, \tilde{s}) \in \mathcal{N}(\tau, \beta)$. This follows the result. 
Lemma 8. Let $(\tilde{x}, \tilde{s}) \in \mathcal{N}(\tau, \beta), G:=L_{\tilde{s}}^{-1} L_{\tilde{x}}$ and $(\ddot{\tilde{x}}, \ddot{\tilde{s}})$ be the solution of system (3.6). Then,

$$
\begin{aligned}
\|\ddot{\tilde{x}} \circ \ddot{\tilde{s}}\|_{F} & \leq \frac{1}{2 \gamma}(\operatorname{cond}(G))^{\frac{3}{2}}(1+\beta \tau)^{2} \mu r^{2}, \\
\operatorname{tr}(\ddot{\tilde{x}} \circ \ddot{\tilde{s}}) & \leq \frac{1}{2 \gamma}(\operatorname{cond}(G))^{\frac{3}{2}}(1+\beta \tau)^{2} \mu r^{2} .
\end{aligned}
$$

Proof. Using Lemma 33 in [17] and Lemmas 4 and 7, it follows

$$
\begin{aligned}
\|\ddot{\tilde{x}} \circ \ddot{\tilde{s}}\|_{F} & \leq\|\ddot{\tilde{x}}\|_{F}\|\ddot{\tilde{s}}\|_{F} \leq \frac{1}{2} \sqrt{\operatorname{cond}(G)}\left(\left\|G^{\frac{-1}{2}} \ddot{\tilde{x}}\right\|_{F}^{2}+\left\|G^{\frac{1}{2}} \ddot{\tilde{s}}\right\|_{F}^{2}\right) \\
& \leq \frac{1}{2} \sqrt{\operatorname{cond}(G)}\left\|G^{\frac{-1}{2}} \ddot{\tilde{x}}+G^{\frac{1}{2}} \ddot{\tilde{s}}\right\|_{F}^{2} \leq \frac{1}{2 \gamma}(\operatorname{cond}(G))^{\frac{3}{2}}(1+\beta \tau)^{2} \mu r^{2}
\end{aligned}
$$

which concludes the inequality (4.4). In the same way as the proof of (4.2), the inequality (4.5) is proved. This ends the proof.

Lemma 9. Let $(\dot{\tilde{x}}, \dot{\tilde{s}})$ and $(\ddot{\tilde{x}}, \ddot{\tilde{s}})$ be the solutions of systems (3.5) and (3.6). Then,

$$
\begin{aligned}
\|\dot{\tilde{x}} \circ \ddot{\tilde{s}}\|_{F} & \leq \frac{1}{\sqrt{\gamma}} \operatorname{cond}(G)(1+\beta \tau)^{\frac{3}{2}} \mu r^{\frac{3}{2}}, \\
\|\dot{\tilde{s}} \circ \ddot{\tilde{x}}\|_{F} & \leq \frac{1}{\sqrt{\gamma}} \operatorname{cond}(G)(1+\beta \tau)^{\frac{3}{2}} \mu r^{\frac{3}{2}} .
\end{aligned}
$$

Proof. Using Lemma 33 in [17] and Lemmas 6 and 8, we have

$$
\begin{aligned}
\|\dot{\tilde{x}} \circ \ddot{\tilde{s}}\|_{F} \leq\|\dot{\tilde{x}}\|_{F}\|\ddot{\tilde{s}}\|_{F} & \leq \sqrt{\operatorname{cond}(G)}\left\|G^{\frac{-1}{2}} \dot{\tilde{x}}\right\|_{F}\left\|G^{\frac{1}{2}} \ddot{\tilde{s}}\right\|_{F} \\
& \leq \frac{1}{\sqrt{\gamma}} \operatorname{cond}(G)(1+\beta \tau)^{\frac{3}{2}} \mu r^{\frac{3}{2}}
\end{aligned}
$$

which implies (4.6). The proof of (4.7) is similar to (4.6) and therefore is omitted.

Using Lemma 4.52 in [6] and Lemma 9, the following corollary is derived.

Corollary 2. Let $x, s \in$ int $\mathcal{K}$ and $(\dot{\tilde{x}}, \dot{\tilde{s}})$ and $(\ddot{\tilde{x}}, \ddot{\tilde{s}})$ be the solutions of systems (3.5) and (3.6). Then,

$$
\begin{aligned}
&|\operatorname{tr}(\dot{\tilde{x}} \circ \ddot{\tilde{s}})| \leq \frac{1}{\sqrt{\gamma}} \operatorname{cond}(G)(1+\beta \tau)^{\frac{3}{2}} \mu r^{\frac{3}{2}} \\
&|\operatorname{tr}(\dot{\tilde{s}} \circ \ddot{\tilde{x}})| \leq \frac{1}{\sqrt{\gamma}} \operatorname{cond}(G)(1+\beta \tau)^{\frac{3}{2}} \mu r^{\frac{3}{2}} .
\end{aligned}
$$

\subsection{Convergence analysis}

In this subsection, we prove the polynomial complexity of Algorithm 1. To this end, we need to obtain a lower bound for the largest step size $\sin \left(\hat{\alpha}^{k}\right)$ such 
that the conditions (3.11) and (3.12) are held. More precisely, it is necessary to obtain a lower bound for the step $\operatorname{size} \sin (\hat{\alpha})$ such that it not only guarantees the reduction of the duality gap $\operatorname{tr}(\tilde{x} \circ \tilde{s})$ but also ensures that the new generated point $(\tilde{x}(\alpha), \tilde{s}(\alpha))$ belongs to $\mathcal{N}(\tau, \beta)$. To this end, let to state the following lemma which plays an important role in our analysis.

Lemma 10. Let $\varphi(\alpha)$ be defined as (3.10). Then

$$
d_{1}(\alpha) \leq \operatorname{tr}(\varphi(\alpha)) \leq d_{2}(\alpha)
$$

where

$$
\begin{aligned}
d_{1}(\alpha) & :=-\frac{1}{2} \sqrt{\operatorname{cond}(G)}(1+\beta \tau) \sin ^{4}(\alpha) \mu r-\frac{2}{\sqrt{\gamma}} \operatorname{cond}(G) \\
& \times(1+\beta \tau)^{\frac{3}{2}} \sin ^{3}(\alpha) \mu r^{\frac{3}{2}}, \quad d_{2}(\alpha):=\frac{2}{\sqrt{\gamma}} \operatorname{cond}(G)(1+\beta \tau)^{\frac{3}{2}} \\
& \times \sin ^{3}(\alpha) \mu r^{\frac{3}{2}}+\frac{1}{2 \gamma}(\operatorname{cond}(G))^{\frac{3}{2}}(1+\beta \tau)^{2} \sin ^{4}(\alpha) \mu r^{2} .
\end{aligned}
$$

Proof. To prove the left hand side inequality in (4.8), using Lemmas 4 and 6 and Corollary 2, we derive

$$
\begin{aligned}
& \operatorname{tr}(\varphi(\alpha))=-g^{2}(\alpha) \operatorname{tr}(\dot{\tilde{x}} \circ \dot{\tilde{s}})-\sin (\alpha) g(\alpha) \operatorname{tr}(\dot{\tilde{x}} \circ \ddot{\tilde{s}}+\dot{\tilde{s}} \circ \ddot{\tilde{x}})+g^{2}(\alpha) \operatorname{tr}(\ddot{\tilde{x}} \circ \ddot{\tilde{s}}) \\
& \quad \geq-g^{2}(\alpha) \operatorname{tr}(\dot{\tilde{x}} \circ \dot{\tilde{s}})-\sin (\alpha) g(\alpha)(\operatorname{tr}(\dot{\tilde{x}} \circ \ddot{\tilde{s}})+\operatorname{tr}(\dot{\tilde{s}} \circ \ddot{\tilde{x}})) \\
& \quad \geq-\frac{1}{2} g^{2}(\alpha) \sqrt{\operatorname{cond}(G)}(1+\beta \tau) \mu r-\frac{2}{\sqrt{\gamma}} \operatorname{cond}(G)(1+\beta \tau)^{\frac{3}{2}} \sin (\alpha) g(\alpha) \mu r^{\frac{3}{2}} \\
& \quad \geq-\frac{1}{2} \sqrt{\operatorname{cond}(G)}(1+\beta \tau) \sin ^{4}(\alpha) \mu r-\frac{2}{\sqrt{\gamma}} \operatorname{cond}(G)(1+\beta \tau)^{\frac{3}{2}} \sin ^{3}(\alpha) \mu r^{\frac{3}{2}}
\end{aligned}
$$

which follows the left-hand side of the inequality in (4.8). On the other hand, we have

$$
\begin{aligned}
& \operatorname{tr}(\varphi(\alpha))=-g^{2}(\alpha) \operatorname{tr}(\dot{\tilde{x}} \circ \dot{\tilde{s}})-\sin (\alpha) g(\alpha) \operatorname{tr}(\dot{\tilde{x}} \circ \ddot{\tilde{s}}+\dot{\tilde{s}} \circ \ddot{\tilde{x}})+g^{2}(\alpha) \operatorname{tr}(\ddot{\tilde{x}} \circ \ddot{\tilde{s}}) \\
& \quad \leq-\sin (\alpha) g(\alpha)(\operatorname{tr}(\dot{\tilde{x}} \circ \ddot{\tilde{s}})+\operatorname{tr}(\dot{\tilde{s}} \circ \ddot{\tilde{x}}))+g^{2}(\alpha) \operatorname{tr}(\ddot{\tilde{x}} \circ \ddot{\tilde{s}}) \\
& \quad \leq \frac{2}{\sqrt{\gamma}} \operatorname{cond}(G)(1+\beta \tau)^{\frac{3}{2}} \sin ^{3}(\alpha) \mu r^{\frac{3}{2}}+\frac{1}{2 \gamma}(\operatorname{cond}(G))^{\frac{3}{2}}(1+\beta \tau)^{2} \sin ^{4}(\alpha) \mu r^{2}
\end{aligned}
$$

which proves the right hand side inequality in (4.8). The proof is complete.

Lemma 11. Let $\sin (\hat{\alpha})$ be the largest step size which satisfies the condition (3.11). Then, $\sin (\hat{\alpha}) \geq \sin \left(\hat{\alpha}^{0}\right)$, where $\sin \left(\hat{\alpha}^{0}\right):=0.5 \beta \tau / \sqrt{\operatorname{rcond}(G)}$.

Proof. Due to (3.9), (3.10) and the definition of $\tilde{r}_{c}$, we have

$$
\begin{aligned}
\mu(\alpha) & =\tilde{\mu}(\alpha)=\frac{1}{r} \operatorname{tr}(\tilde{x}(\alpha) \circ \tilde{s}(\alpha))=\frac{1}{r}\left(\operatorname{tr}(\tilde{x} \circ \tilde{s})-\sin (\alpha) \operatorname{tr}\left(\tilde{r}_{c}\right)+\operatorname{tr}(\varphi(\alpha))\right) \\
& =\mu+\sin (\alpha)\left(\tau \mu-\mu+\frac{\sqrt{r}-1}{r} \operatorname{tr}(\tau \mu e-\tilde{x} \circ \tilde{s})^{+}\right)+\frac{\operatorname{tr}(\varphi(\alpha))}{r} \\
& \leq \mu+\sin (\alpha)\left(\tau \mu-\mu+\frac{\sqrt{r}-1}{\sqrt{r}}\left\|(\tau \mu e-\tilde{x} \circ \tilde{s})^{+}\right\|_{F}\right)+\frac{d_{2}(\alpha)}{r} .
\end{aligned}
$$


Thus, using the definition of $\mathcal{N}(\tau, \beta)$ and Lemma 10, we derive

$$
\begin{aligned}
& \mu(\alpha) \leq \mu+(\tau-1+\beta \tau) \sin (\alpha) \mu+\frac{1}{r} d_{2}(\alpha)=\mu+(\tau-1+\beta \tau) \sin (\alpha) \mu \\
& \quad+\frac{2}{\sqrt{\gamma}} \operatorname{cond}(G)(1+\beta \tau)^{\frac{3}{2}} \sin ^{3}(\alpha) \mu \sqrt{r}+\frac{1}{2 \gamma}(\operatorname{cond}(G))^{\frac{3}{2}}(1+\beta \tau)^{2} \sin ^{4}(\alpha) \mu r \\
& \leq\left[1-\left[1-\tau-\beta \tau-\frac{2}{\sqrt{\gamma}} \operatorname{cond}(G)(1+\beta \tau)^{\frac{3}{2}} \sin ^{2}\left(\hat{\alpha}^{0}\right) \sqrt{r}\right.\right. \\
&\left.\left.\quad-\frac{1}{2 \gamma}(\operatorname{cond}(G))^{\frac{3}{2}}(1+\beta \tau)^{2} \sin ^{3}\left(\hat{\alpha}^{0}\right) r\right] \sin (\alpha)\right] \mu \\
& \leq {\left[1-\left[1-\frac{1}{4}-\frac{1}{8}-\frac{1}{2 \sqrt{\gamma r}}(1+\beta \tau)^{\frac{3}{2}} \beta^{2} \tau^{2}-\frac{1}{16 \gamma \sqrt{r}}(1+\beta \tau)^{2} \beta^{3} \tau^{3}\right] \sin (\alpha)\right] \mu } \\
& \leq {\left[1-\left[\frac{5}{8}-\frac{1}{\sqrt{6}}(1+\beta \tau)^{\frac{3}{2}} \beta^{2} \tau^{\frac{3}{2}}-\frac{1}{8 \sqrt{3}}(1+\beta \tau)^{2} \beta^{3} \tau^{2}\right] \sin (\alpha)\right] \mu } \\
& \leq {[1-(0.625-0.015-0.001) \sin (\alpha)] \mu \leq\left(1-\frac{1}{2} \sin (\alpha)\right) \mu, }
\end{aligned}
$$

where the third inequality is due to $\sin \left(\hat{\alpha}^{0}\right):=\frac{\beta \tau}{2 \sqrt{r \operatorname{cond}(G)}}$ and the last three inequalities follow from the facts $\frac{1}{\sqrt{\gamma}} \leq \frac{\sqrt{2}}{\sqrt{\tau}}, r \geq 3, \tau \leq \frac{1}{4}$ and $\beta \leq \frac{1}{2}$. This implies that $\sin \left(\hat{\alpha}^{0}\right)$ is a lower bound for the largest step size $\sin (\hat{\alpha})$ which satisfies the condition (3.11). The result is derived.

Lemma 12. Let $\sin (\hat{\alpha}) \geq \sin \left(\hat{\alpha}^{0}\right)$, where $\sin \left(\hat{\alpha}^{0}\right):=\frac{\beta \tau}{2 \sqrt{\operatorname{rcond}(G)}}$. Then, for $\sin (\alpha) \in[0, \sin (\hat{\alpha})]$,

$$
\tilde{\mu}(\alpha) \geq(1-\sin (\alpha)) \mu
$$

Proof. Using (3.9) and Lemma 10, we conclude

$$
\begin{aligned}
& \operatorname{tr}(\tilde{x}(\alpha) \circ \tilde{s}(\alpha))=\operatorname{tr}(\tilde{x} \circ \tilde{s})-\sin (\alpha) \operatorname{tr}\left(\tilde{r}_{c}\right)+\operatorname{tr}(\varphi(\alpha)) \\
& \quad=r \mu+\sin (\alpha)\left(\tau r \mu-r \mu+(\sqrt{r}-1) \operatorname{tr}(\tau \mu e-\tilde{x} \circ \tilde{s})^{+}\right)+\operatorname{tr}(\varphi(\alpha)) \\
& \quad \geq(1-\sin (\alpha)) r \mu+\sin (\alpha) \tau r \mu+d_{1}(\alpha) .
\end{aligned}
$$

On the other hand, we have

$$
\begin{aligned}
& \sin (\alpha) \tau r \mu+d_{1}(\alpha)=\sin (\alpha) \tau r \mu-\frac{1}{2} \sqrt{\operatorname{cond}(G)}(1+\beta \tau) \sin ^{4}(\alpha) \mu r \\
& \quad-\frac{2}{\sqrt{\gamma}} \operatorname{cond}(G)(1+\beta \tau)^{\frac{3}{2}} \sin ^{3}(\alpha) \mu r^{\frac{3}{2}} \geq \sin (\alpha) r \mu \\
& \quad \times\left[\tau-\frac{\sqrt{\operatorname{cond}(G)}}{2}(1+\beta \tau) \sin ^{3}\left(\hat{\alpha}_{0}\right)-\frac{2}{\sqrt{\gamma}} \operatorname{cond}(G)(1+\beta \tau)^{\frac{3}{2}} \sin ^{2}\left(\hat{\alpha}_{0}\right) \sqrt{r}\right] \\
& \geq \sin (\alpha) r \mu\left[\tau-\frac{1}{2}(1+\beta \tau) \frac{\beta^{3} \tau^{3}}{8 \operatorname{cond}(G) r^{\frac{3}{2}}}-2 \sqrt{2}(1+\beta \tau)^{\frac{3}{2}} \frac{\beta^{2} \tau^{\frac{3}{2}}}{4 \sqrt{r}}\right] \geq 0
\end{aligned}
$$


where the last inequality holds for $r \geq 3$ and it follows from $\tau \in\left(0, \frac{1}{4}\right]$ and $\beta \leq \frac{1}{2}$. Substituting (4.11) into (4.10), one has $\tilde{\mu}(\alpha) \geq(1-\sin (\alpha)) \mu$, which concludes the result and ends the proof.

The following lemma is directly used in the proof of Lemma 14

Lemma 13. Let $\tilde{\mu}(\alpha)>0$ and $\sin \left(\hat{\alpha}^{0}\right):=\frac{\beta \tau}{2 \sqrt{r \operatorname{cond}(G)}}$. Then, for all step size $\sin (\alpha) \in\left[0, \sin \left(\hat{\alpha}^{0}\right)\right]$, one has

$$
\left\|\left(\tau \tilde{\mu}(\alpha) e-\tilde{x} \circ \tilde{s}+\sin (\alpha) \tilde{r}_{c}\right)^{+}\right\|_{F} \leq(1-\sin (\alpha) \sqrt{r}) \beta \tau \mu .
$$

Proof. Due to Lemma 11, we have $\mu(\alpha) \leq \mu$. Therefore,

$$
\begin{aligned}
& \left\|\left(\tau \tilde{\mu}(\alpha) e-\tilde{x} \circ \tilde{s}+\sin (\alpha) \tilde{r}_{c}\right)^{+}\right\|_{F} \leq\left\|\left(\tau \mu e-\tilde{x} \circ \tilde{s}+\sin (\alpha) \tilde{r}_{c}\right)^{+}\right\|_{F} \\
& \quad \leq\left\|\left[(1-\sin (\alpha))(\tau \mu e-\tilde{x} \circ \tilde{s})^{-}+(1-\sin (\alpha) \sqrt{r})(\tau \mu e-\tilde{x} \circ \tilde{s})^{+}\right]^{+}\right\|_{F} \\
& \quad \leq\left\|\left[(1-\sin (\alpha) \sqrt{r})(\tau \mu e-\tilde{x} \circ \tilde{s})^{+}\right]^{+}\right\|_{F} \\
& \quad=(1-\sin (\alpha) \sqrt{r})^{+}\left\|(\tau \mu e-\tilde{x} \circ \tilde{s})^{+}\right\|_{F} \leq(1-\sin (\alpha) \sqrt{r}) \beta \tau \mu,
\end{aligned}
$$

where the third inequality follows from Lemma 5.7 in [18] and the last one is due to the facts $(\tilde{x}, \tilde{s}) \in \mathcal{N}(\tau, \beta)$ and $\sin \left(\hat{\alpha}^{0}\right) \leq \frac{1}{\sqrt{r}}$. This completes the proof.

Now, we are ready to present the main result of the paper.

Lemma 14. Let $\sin \left(\hat{\alpha}^{0}\right):=0.5 \beta \tau / \sqrt{\operatorname{rcond}(G)}$ and $(\tilde{x}(\alpha), \tilde{s}(\alpha))$ be defined as in Lemma 1. Then, for all $\sin (\alpha) \in\left[0, \sin \left(\hat{\alpha}^{0}\right)\right]$

$$
(\tilde{x}(\alpha), \tilde{s}(\alpha)) \in \mathcal{N}(\tau, \beta) \text {. }
$$

Proof. To prove the lemma, we need to show that $(\tilde{x}(\alpha), \tilde{s}(\alpha)) \in \mathcal{F}^{0}$ and also $\left\|\left(\tau \tilde{\mu}(\alpha) e-Q_{\tilde{x}(\alpha)^{\frac{1}{2}}} \tilde{s}(\alpha)\right)^{+}\right\|_{F} \leq \beta \tau \tilde{\mu}(\alpha)$. To this end, using Lemma 5.17 in [18], we have

$$
\begin{aligned}
& \left\|(\tau \tilde{\mu}(\alpha) e-\tilde{x}(\alpha) \circ \tilde{s}(\alpha))^{+}\right\|_{F}=\left\|\left(\tau \tilde{\mu}(\alpha) e-\tilde{x} \circ \tilde{s}+\sin (\alpha) \tilde{r}_{c}-\varphi(\alpha)\right)^{+}\right\|_{F} \\
& \quad \leq\left\|\left(\tau \tilde{\mu}(\alpha) e-\tilde{x} \circ \tilde{s}+\sin (\alpha) \tilde{r}_{c}\right)^{+}\right\|_{F}+\left\|(\varphi(\alpha))^{-}\right\|_{F} \\
& \quad \leq\left\|\left(\tau \tilde{\mu}(\alpha) e-\tilde{x} \circ \tilde{s}+\sin (\alpha) \tilde{r}_{c}\right)^{+}\right\|_{F}+\|\varphi(\alpha)\|_{F} \cdot
\end{aligned}
$$

Thus, $\left\|(\tau \tilde{\mu}(\alpha) e-\tilde{x}(\alpha) \circ \tilde{s}(\alpha))^{+}\right\|_{F} \leq \beta \tau \tilde{\mu}(\alpha)$, if

$$
\left\|\left(\tau \tilde{\mu}(\alpha) e-\tilde{x} \circ \tilde{s}+\sin (\alpha) \tilde{r}_{c}\right)^{+}\right\|_{F}+\|\varphi(\alpha)\|_{F} \leq \beta \tau \tilde{\mu}(\alpha) .
$$


Using Lemmas 6, 8 and 9, it follows

$$
\begin{aligned}
&\|\varphi(\alpha)\|_{F}=\left\|-g^{2}(\alpha) \dot{\tilde{x}} \circ \dot{\tilde{s}}-\sin (\alpha) g(\alpha)(\dot{\tilde{x}} \circ \ddot{\tilde{s}}+\dot{\tilde{s}} \circ \ddot{\tilde{x}})+g^{2}(\alpha) \ddot{\tilde{x}} \circ \ddot{\tilde{s}}\right\|_{F} \\
& \leq \sin ^{4}(\alpha)\|\dot{\tilde{x}} \circ \dot{\tilde{s}}\|_{F}+\sin ^{3}(\alpha)\left(\|\dot{\tilde{x}} \circ \ddot{\tilde{s}}\|_{F}+\|\dot{\tilde{s}} \circ \ddot{\tilde{x}}\|_{F}\right)+\sin ^{4}(\alpha)\|\ddot{\tilde{x}} \circ \ddot{\tilde{s}}\|_{F} \\
& \leq \frac{1}{2} \sqrt{\operatorname{cond}(G)}(1+\beta \tau) \sin ^{4}(\alpha) \mu r+\frac{2}{\sqrt{\gamma}} \operatorname{cond}(G)(1+\beta \tau)^{\frac{3}{2}} \sin ^{3}(\alpha) \mu r^{\frac{3}{2}} \\
&+\frac{1}{2 \gamma}(\operatorname{cond}(G))^{\frac{3}{2}}(1+\beta \tau)^{2} \sin ^{4}(\alpha) \mu r^{2} \\
& \leq \sin (\alpha) \sqrt{r} \mu\left[\frac{1}{2} \sqrt{\operatorname{cond}(G)}(1+\beta \tau) \sin ^{3}\left(\hat{\alpha}^{0}\right) \sqrt{r}+\frac{2}{\sqrt{\gamma}} \operatorname{cond}(G)\right. \\
&\left.\times(1+\beta \tau)^{\frac{3}{2}} \sin ^{2}\left(\hat{\alpha}^{0}\right) r+\frac{1}{2 \gamma}(\operatorname{cond}(G))^{\frac{3}{2}}(1+\beta \tau)^{2} \sin ^{3}\left(\hat{\alpha}^{0}\right) r^{\frac{3}{2}}\right] \\
& \leq \sin (\alpha) \sqrt{r} \beta \tau \mu\left[\frac{1}{16 r} \frac{1}{\operatorname{cond}(G)}(1+\beta \tau) \beta^{2} \tau^{2}+\frac{\sqrt{2}}{2}(1+\beta \tau)^{\frac{3}{2}} \beta \sqrt{\tau}\right. \\
&\left.+\frac{1}{8}(1+\beta \tau)^{2} \beta^{2} \tau\right] \leq \frac{22}{100} \sin (\alpha) \sqrt{r} \beta \tau \mu,
\end{aligned}
$$

where the last inequality follows from $r \geq 3, \tau \leq \frac{1}{4}, \beta \leq \frac{1}{2}$ and $\operatorname{cond}(G) \geq 1$.

Now, using Lemma 12, Lemma 13 and (4.15), we derive

$$
\begin{aligned}
& \left\|\left(\tau \mu(\alpha) e-x s+\sin (\alpha) r_{c}\right)^{+}\right\|+\|\varphi(\alpha)\|-\beta \tau \mu(\alpha) \\
& \quad \leq(1-\sin (\alpha) \sqrt{r}) \beta \tau \mu+\frac{22}{100} \sin (\alpha) \sqrt{r} \beta \tau \mu-(1-\sin (\alpha)) \beta \tau \mu \\
& \quad=\sin (\alpha) \sqrt{r} \beta \tau \mu\left(-1+\frac{22}{100}+\frac{1}{\sqrt{r}}\right) \leq 0,
\end{aligned}
$$

which implies inequality (4.14). Therefore

$$
\left\|(\tau \tilde{\mu}(\alpha) e-\tilde{x}(\alpha) \circ \tilde{s}(\alpha))^{+}\right\|_{F} \leq \beta \tau \tilde{\mu}(\alpha) .
$$

On the other hand, due to (4.16) and Lemma 12, we have

$$
\lambda_{i}(\tilde{x}(\alpha) \circ \tilde{s}(\alpha)) \geq(1-\beta) \tau \tilde{\mu}(\alpha) \geq(1-\beta)(1-\sin (\alpha)) \tau \mu>0,
$$

which implies $\tilde{x}(\alpha) \circ \tilde{s}(\alpha) \in$ int $\mathcal{K}$ and therefore, using Lemma 2.15 in [23], $\operatorname{det}(\tilde{x}(\alpha)) \neq 0$ and $\operatorname{det}(\tilde{s}(\alpha)) \neq 0$ for all $\alpha \in\left[0, \frac{\pi}{2}[\right.$. Furthermore, since $\tilde{x} \in$ int $\mathcal{K}_{\text {and }} \tilde{s} \in$ int $\mathcal{K}$, by the continuity, it follows that both $\tilde{x}(\alpha)$ and $\tilde{s}(\alpha)$ belong to int $\mathcal{K}$ for $\alpha \in\left[0, \frac{\pi}{2}[\right.$. Moreover, since $\tilde{s}(\alpha)=\tilde{M} \tilde{x}(\alpha)+\tilde{q}$ it follows that $(\tilde{x}(\alpha), \tilde{s}(\alpha)) \in \mathcal{F}^{0}$.

Finally, using Lemma 5.10 in [12] and (4.16), we conclude

$$
\left\|\left(\tau \tilde{\mu}(\alpha) e-Q_{\tilde{x}(\alpha)^{\frac{1}{2}}} \tilde{s}(\alpha)\right)^{+}\right\|_{F} \leq\left\|(\tau \tilde{\mu}(\alpha) e-\tilde{x}(\alpha) \circ \tilde{s}(\alpha))^{+}\right\|_{F} \leq \beta \tau \tilde{\mu}(\alpha) .
$$

This result is concluded. 


\subsection{Iteration bound}

Now, we are ready to compute the complexity bound of Algorithm 1. The following lemma tasks this goal.

Lemma 15. Let $\sqrt{\operatorname{cond}(G)}$ be bounded above by $\kappa<\infty$ for all iterations, $(x, s) \in \mathcal{N}(\tau, \beta)$ and $\sin \left(\hat{\alpha}^{0}\right):=0.5 \beta \tau / \sqrt{\operatorname{rcond}(G)}$. Then, Algorithm 1 will terminate in at most $O\left(\kappa \sqrt{r} \log \varepsilon^{-1}\right)$ iterations.

Proof. Let $\sin \left(\hat{\alpha}^{0}\right):=0.5 \beta \tau / \sqrt{r \operatorname{cond}(G)}$. Then, due to (3.11), we have

$$
\begin{aligned}
\mu\left(\hat{\alpha}^{k+1}\right)=\tilde{\mu}\left(\hat{\alpha}^{k+1}\right) & \leq \prod_{i=1}^{k}\left(1-\frac{1}{2} \sin \left(\hat{\alpha}^{i}\right)\right) \mu^{0} \leq\left(1-\frac{1}{2} \sin \left(\hat{\alpha}^{0}\right)\right)^{k} \mu^{0} \\
& =\left(1-\frac{1}{4} \frac{\beta \tau}{\sqrt{r \operatorname{cond}(G)}}\right)^{k} \mu^{0} \leq\left(1-\frac{1}{4} \frac{\beta \tau}{\kappa \sqrt{r}}\right)^{k} \mu^{0},
\end{aligned}
$$

which implies

$$
\left(1-\frac{1}{4} \frac{\beta \tau}{\kappa \sqrt{r}}\right)^{k} \mu^{0} \leq \varepsilon,
$$

for $k \geq\left(4 \kappa \sqrt{r} \log \varepsilon^{-1}\right) /(\beta \tau)$. Therefore, Algorithm 1 will terminate after at most $\left\lceil 4 \kappa \sqrt{r} \log \varepsilon^{-1}\right\rceil$ iterations. This completes the proof.

The following lemma gives a bound on $\operatorname{cond}(\mathbf{G})$ for some specific search directions.

Lemma 16 [Lemma 36 in [17]]. For the $N T$ direction, $\operatorname{cond}(\boldsymbol{G})=1$ while for the $x s$ and $s x$ directions, $\operatorname{cond}(\boldsymbol{G}) \leq r / \gamma$.

Using the bounds on $\sqrt{\operatorname{cond}(G)}$ in Lemma 16 for the NT, $x s$ and $s x$ search directions, we have the following iteration complexities.

Corollary 3. If the NT search direction is used, the iteration complexity of Algorithm 1 is $O\left(\sqrt{r} \log \varepsilon^{-1}\right)$. If the $x s$ and $s x$ search directions are used, the iteration complexities of Algorithm 1 are $O\left(r \log \varepsilon^{-1}\right)$.

\section{Concluding remarks}

This paper proposed an arc search feasible interior-point algorithm for monotone SCLCPs. The algorithm follows an ellipsoidal approximation of the central path and generates a sequence of iterates in a wide neighborhood of the central path. For a commutative class of search directions which includes NT-search direction and the $x s$ and $s x$ directions, the convergence analysis of the algorithm was proved. Moreover, we derived the complexity bound of the algorithm and proved that it coincides with the currently best known theoretical complexity bounds for feasible interior-point algorithm. 


\section{Acknowledgement}

The first three authors thank Shahrekord University for the financial support. These authors were also partially supported by the Center of Excellence for Mathematics, University of Shahrekord, Shahrekord, Iran. The second and fourth authors thank Ryerson University for the financial support.

\section{References}

[1] W. Ai and S.Z Zhang. An $o(\sqrt{n} l)$ iteration primal-dual path-following method, based on wide neighborhoods and large updates, for monotone LCP. SIAM J. Optim, 16(2):400-417, 2005. https://doi.org/10.1137/040604492.

[2] M.P. Carmo. Differential Geometry of Curves and Surfaces. Prentice-Hall, New Jersey, 1976.

[3] J. Faraut and A. Korányi. Analysis on Symmetric Cones. Oxford University Press, Oxford, UK, 1994.

[4] L. Faybusovich. Euclidean Jordan algebras and interior-point algorithms. Positivity, 1(4):331-357, 1997. https://doi.org/10.1023/A:1009701824047.

[5] M.S Gowda and R. Sznajder. Some global uniquness and solvability results for linear complementarity problems over symmetric cones. SIAM J. Optim, 18(2):461-481, 2007. https://doi.org/10.1137/06065943X.

[6] G. Gu. Full-step interior point methods for symmetric optimization. Faculty of Mathematics and Computer Science, TU Delft, NL-2628 CD Delft, The Netherlands, 2009.

[7] N. Karmarkar. A new polynomial-time algorithm for linear programming. Proceedings of the 16th Annual ACM Symposium on Theory of Computing, pp. 302-311, 1984. https://doi.org/10.1145/800057.808695.

[8] B. Kheirfam and N. Mahdavi-Amiri. A new interior-point algorithm based on modified Nesterov-Todd direction for symmetric cone linear complementarity problem. Optim. Lett., 8(3):1017-1029, 2014. https://doi.org/10.1007/s11590013-0618-5.

[9] B. Kheirfam and N. Mahdavi-Amiri. An infeasible interior-point algorithm based on modified Nesterov and Todd directions for symmetric linear complementarity problem. Optimization, 64(7):1577-1591, 2015. https://doi.org/10.1080/02331934.2013.869877.

[10] Y. Li and T. Terlaky. A new class of large neighborhood pathfollowing interior point algorithms for semidefinite optimization with $o\left(\sqrt{n} \log \left(\frac{\operatorname{tr}\left(x^{0} s^{0}\right)}{\varepsilon}\right)\right)$ iteration complexity. SIAM J. Optim, 20(6):2853-2875, 2010. https://doi.org/10.1137/080729311.

[11] Y. Lim. Geometric means on symmetric cones. Arch. Math., 75(1):39-56, 2000. https://doi.org/10.1007/s000130050471.

[12] C. Liu. Study on complexity of some interior-point algorithms in conic programming. Xixian University, 2012.

[13] X. Yang, H. Liu and Y. Zhang. A new strategy in the complexity analysis of an infeasible-interior-point method for symmetric cone programming. J. Optim. Theory Appl., 166(2):572-587, 2015. https://doi.org/10.1007/s10957-014-0670$\mathrm{z}$. 
[14] X. Yang, H. Liu and Y. Zhang. An arc-search infeasible-interior-point method for symmetric optimization in a wide neighborhood of the central path. Optim. Lett., 11(1):135-152, 2016. https://doi.org/10.1007/s11590-016-0997-5.

[15] B.K. Rangarajan. Polynomial convergence of infeasible-interior-point methods over symmetric cones. SIAM J. Optim., 16(4):1211-1229, 2006. https://doi.org/10.1137/040606557.

[16] C. Roos. A full-Newton step $O(n)$ infeasible interior-point algorithm for linear optimization. SIAM J. Optim., 16(2):1110-1136, 2006. https://doi.org/10.1137/050623917.

[17] S.H. Schmieta and F. Alizadeh. Extension of primal-dual interior-point algorithms to symmetric cones. Math. Program. Ser A, 96(3):409-438, 2003. https://doi.org/10.1007/s10107-003-0380-z.

[18] H. Liu, X. Yang and C. Liu. A new wide neighborhood primal-dual infeasibleinterior-point method for symmetric cone programming. J. Optim. Theory Appl., 158(3):796-815, 2013. https://doi.org/10.1007/s10957-013-0303-y.

[19] Y. Yang. A polynomial arc-search interior-point algorithm for convex quadratic programming. Eur. J. Oper. Res., 215(1):25-38, 2011. https://doi.org/10.1016/j.ejor.2011.06.020.

[20] Y. Yang. A polynomial arc-search interior-point algorithm for linear programming. J. Optim. Theory Appl., 158(3):859-873, 2013. https://doi.org/10.1007/s10957-013-0281-0.

[21] Y. Yoshise. Interior-point trajectories and a homogeneous model for nonlinear complementarity problems over symmetric cones. SIAM J. Optim., 17(4):11291153, 2006. https://doi.org/10.1137/04061427X.

[22] G.Q. Wang, M. Li, Y. Yue and X. Cai. New complexity analysis of interior-point methods for the Cartesian $p_{*}(\kappa)$-SCLCP. J. Ineq. Appl., 285:1-23, 2013.

[23] G. Gu, M. Zangiabadi and C. Roos. Full Nesterov-Todd step infeasible interiorpoint method for symmetric optimization. European J. Oper. Res., 214(3):473484, 2011. https://doi.org/10.1016/j.ejor.2011.02.022.

[24] M. Pirhaji, M. Zangiabadi and H. Mansouri. An $\ell_{2}$-neighborhood infeasible interior-point algorithm for linear complementarity problems. 4OR-Q. J. Oper. Res., 15(2):111-131, 2016. https://doi.org/10.1007/s10288-016-0325-z.

[25] X. Yang, Y. Zhang and H. Liu. A wide neighborhood infeasible-interior-point method with arc-search for linear programming. J. Appl. Math. Comput., 51(12):209-225, 2016. https://doi.org/10.1007/s12190-015-0900-z. 